\title{
A multicenter, randomized, double-blind, placebo-controlled study to evaluate the efficacy of immunosuppression in biopsy-proven virus-negative myocarditis or inflammatory cardiomyopathy (IMPROVE-MC)
}

Krzysztof Ozierański ${ }^{1}$, Agata Tymińska ${ }^{1}$, Michal Marchel ${ }^{1}$, Łukasz Januszkiewicz ${ }^{1}$, Cezary Maciejewski ${ }^{1}$, Renata Główczyńska ${ }^{1}$, Renzo Marcolongo ${ }^{2,3}$, Alida L.P. Caforio ${ }^{2}$,

Romuald Wojnicz ${ }^{4}$, Katarzyna Mizia-Stec ${ }^{5}$, Jacek Grzybowski ${ }^{6}$, Mariusz Gąsior ${ }^{7}$,

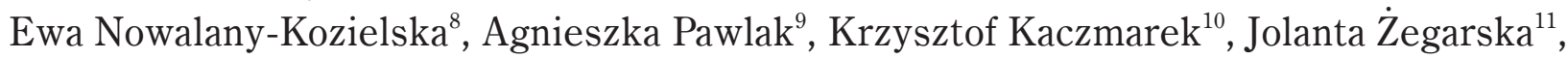
Leszek Pączek ${ }^{11}$, Paweł Balsam ${ }^{1}$, Grzegorz Opolski ${ }^{1}$, Marcin Grabowski ${ }^{1}$

${ }^{1} 1^{\text {st }}$ Department of Cardiology, Medical University of Warsaw, Poland

${ }^{2}$ Division of Cardiology, Department of Cardiac Thoracic Vascular Sciences and Public Health, University of Padova, Italy

${ }^{3}$ Hematology and Clinical Immunology Unit and Cardio-immunology outpatient Clinic, University of Padova, Italy

${ }^{4}$ Department of Histology and Cell Pathology in Zabrze, School of Medicine with the Division of Dentistry,

Medical University of Silesia in Katowice, Poland

${ }^{5} 1^{\text {st }}$ Department of Cardiology, School of Medicine in Katowice,

Medical University of Silesia, Katowice, Poland

${ }^{6}$ Department of Cardiomyopathy, National Institute of Cardiology, Warsaw, Poland

${ }^{7} 3^{\text {rd }}$ Department of Cardiology, Faculty of Medical Sciences in Zabrze,

Medical University of Silesia, Katowice, Poland

${ }^{8} 2^{\text {nd }}$ Department of Cardiology, Faculty of Medical Sciences in Zabrze,

Medical University of Silesia, Katowice, Poland

${ }^{9}$ Department of Invasive Cardiology, Central Clinical Hospital of the Ministry

of the Interior and Administration, Warsaw, Poland

${ }^{10}$ Department of Electrocardiology, Central University Hospital, Medical University of Lodz, Poland

${ }^{11}$ Department of Immunology, Transplant Medicine and Internal Medicine,

Medical University of Warsaw, Poland

\section{Introduction}

\section{Background and rationale}

Myocarditis is defined as an inflammatory heart disease and is an important cause of dilated cardiomyopathy, acute heart failure (HF), life- threatening arrythmias and sudden death [1-5]. It is estimated to affect thousands of adults and children around the world each year generating serious medical and social problems. Post-mortem studies of sudden death in the young report a highly variable prevalence of active myocarditis, even up to $42 \%$ of

Address for correspondence: Agata Tymińska, MD, PhD, First Department of Cardiology, Medical University of Warsaw, ul. Banacha 1a, 02-097 Warszawa, Poland, tel: +48 22 5992958, fax: +48 22 5991957, e-mail: agata.tyminska@wum.edu.pl 
cases [6, 7]. Moreover, myocarditis was confirmed in $9-16 \%$ of adults with unexplained non-ischemic dilated cardiomyopathy in endomyocardial biopsy (EMB) $[8,9]$. Importantly, more than 300,000 patients die each year worldwide from dilated cardiomyopathy caused by myocarditis, while 5 -year mortality in the course of dilated cardiomyopathy is $20-50 \%[10,11]$. What is more, in the period from 1990 to 2015 an increase in morbidity and mortality from myocarditis was recorded [11].

Diagnosis of clinically suspected myocarditis is demanding and based on the clinical picture (signs and symptoms) and deviations in additional tests (electrocardiogram [ECG] abnormalities, functional and/or structural disorders in echocardiography and/or cardiac magnetic resonance [CMR], elevated biomarkers of myocardial necrosis) [1]. The more criteria that are fulfilled, the higher the likelihood of myocarditis. According to the European Society of Cardiology (ESC) position statement, myocarditis is determined by histologic, immunologic and immunohistochemical confirmation of clinically suspected myocarditis. Therefore, although still infrequently performed, EMB is a gold standard for confirmation of ongoing myocarditis [1]. Inflammatory cardiomyopathy is defined as myocarditis with systolic or diastolic cardiac dysfunction [1].

Based on the pathophysiology of inflammatory processes occurring in the course of myocarditis, it is assumed that the disease-specific treatment should include immunosuppressive therapy. However, current recommendations of the ESC Working Group on Myocardial and Pericardial Diseases weakly support the use of immunosuppressive therapy in virus-negative myocarditis or inflammatory cardiomyopathy refractory to standard therapy in patients with no contraindications to immunosuppression due to the lack of high-quality data [1]. There is, however, a general international agreement that specific treatment is reserved for those with the most severe and life-threatening clinical presentation (acute dilated cardiomyopathy caused by giant cell myocarditis, cardiac sarcoidosis and eosinophilic myocarditis) [1, 10, 12, 13]. Moreover, according to a current dogma, when virus particles/genome is present in myocardial tissue immunosuppression is contraindicated [1]. The current recommendations for myocarditis indicate a significant need for a well-planned, multicenter, randomized, placebo-controlled trial to confirm the efficacy and safety of immunosuppressive therapy for myocarditis, based on the diagnosis made with EMB $[1,14]$.
Among the immunosuppressive agents, mainly steroids and azathioprine (frequently in combination) have been tested $[1,12,13]$. Prednisone is a well-known corticosteroid used extensively due to its anti-inflammatory and immunosuppressive properties. Prednisone reduces the number of lymphocytes, eosinophils, monocytes (whereas neutrophil counts increase), reduces the accumulation of leukocytes and their adhesion to the endothelium, inhibits the phagocytosis process and lysosomal breakdown, and blocks IgE-dependent secretion of histamine and leukotrienes. Azathioprine (1-methyl-4-nitro-5-imidazolyl derivative of thioguanine) is an immunosuppressive agent used for the treatment of rheumatic diseases (such as rheumatoid arthritis, systemic lupus erythematosus, dermatomyositis and polymyositis, systemic sclerosis, and systemic vasculitis), inflammatory bowel disease and for the prevention of organ transplant rejection [15]. The mechanism of action of azathioprine is not fully understood, it probably blocks sulfhydryl groups by alkylation. Owing to the release of 6-mercaptopurine, it acts as an antimetabolite for purine bases, leads to disturbance of deoxyribonucleic acid (DNA) synthesis by incorporating purine thioanalogues into the DNA chain, and consequently inhibits the biosynthesis of nucleic acids and prevents the proliferation of participating cells in the immune response [15-17].

\section{Objectives}

Based on the current knowledge, the objective of this trial is to assess the efficacy and safety of a 12 -month treatment with prednisone and azathioprine compared to placebo on top of guideline-recommended medical therapy in patients with biopsy-proven virus-negative myocarditis or inflammatory cardiomyopathy and reduced left ventricular ejection fraction ( $\mathrm{LVEF} \leq 45 \%)$. The study will also assess the maintenance of the therapy effects after a subsequent 12-month observation period. In addition, the trial aims to strengthen the position of EMB and implement the proposed diagnostic and therapeutic processes on a broad scale.

\section{Study design}

The study is an investigator-initiated, randomized, phase IV, double-blind, placebo-controlled, parallel-group trial with an allocation ratio of $1: 1$ to either prednisone and azathioprine or placebo matching prednisone and placebo matching azathioprine. The estimated length of the double-blind 


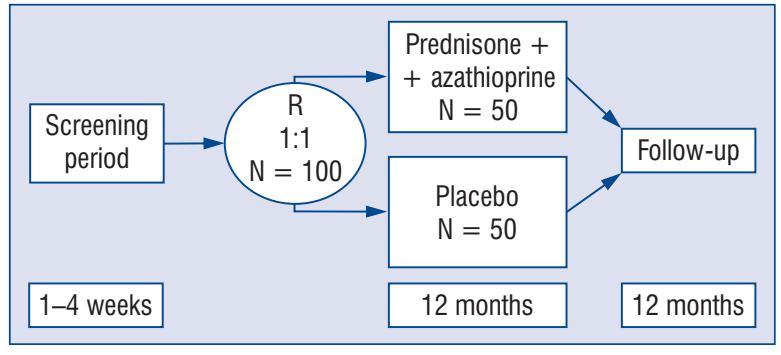

Figure 1. Study design; R - randomization.

treatment will consist of 12 months for each patient. After the 12-month active treatment period, the patients will enter a 12-month follow-up period. The trial design is shown in Figure 1.

The study was registered in ClinicalTrials.gov (NCT04654988) and EudraCT (2020-003877-23). The study protocol conforms to the Standard Protocol Items: Recommendations for Interventional Trials (SPIRIT) 2013 statement [18].

\section{Participants}

A total of 100 individuals (both men and women), aged 18-70 years old, with EMB-confirmed myocarditis or inflammatory cardiomyopathy, with $\mathrm{LVEF} \leq 45 \%$ and $\geq 3$-months history of symptoms will be enrolled in the study. To be eligible for inclusion in this study, patients must fulfill all of the inclusion, and none of the exclusion criteria (Table 1).

\section{Intervention}

Prednisone (Encorton $\left.{ }^{\circledR}\right) 5$ and $10 \mathrm{mg}$ oral tablets and azathioprine (Azathioprine VIS $\left.{ }^{\circledR}\right) 50 \mathrm{mg}$ oral tablets versus placebo (matching prednisone and matching azathioprine) on top of standard medical treatment.

Prednisone/placebo matching prednisone dosing: $1 \mathrm{mg} / \mathrm{kg}$ daily for 2 weeks followed by a gradually tapered dose up to 6 months.

Azathioprine/placebo matching azathioprine dosing: $2 \mathrm{mg} / \mathrm{kg}$ daily for 12 months.

Compliance with the treatment will be monitored and promoted during each study visit to improve adherence to the protocol. Reasons for temporal or permanent treatment discontinuation are depicted in Table 2.

Every attempt should be made to maintain patients on the target study drug(s) dose levels throughout the trial. The investigator may consider down-titrating doses, depending on the tolerance and adverse events (AEs) (i.e., moderate-severe gastro-intestinal intolerance; uncontrolled diabe- tes; renal, pancreas, or liver dysfunction; clinically notable laboratory abnormalities [hematology, blood chemistry]).

\section{Trial setting}

Drawing on feasibility evaluations already performed, patients will be recruited in seven tertiary centers (mostly academic centers) experienced in clinical management (including performing EMB) of patients with HF. The study will consist of 13 visits: screening, randomization, 8 control visits throughout the active treatment period, and 3 control visits within the 1-year follow-up period (visits and assessments schedule are presented in Table 3). During control visits, changes in a clinical and biochemical status compared to the baseline will be monitored. Clinical assessment will include standard physical examination, the New York Heart Association (NYHA) functional classification, 6-minute walk test, quality of life questionnaires, echocardiography, 12-lead ECG and $48 \mathrm{~h}$ Holter-ECG (frequency according to the protocol). CMR and EMB will be performed during screening and after 1-year of treatment. All study participants will be recommended moderate intensity physical activity defined as an individual being able to comfortably maintain conversation during exertion [19]. Higher intensity physical activity will be discouraged

\section{Description of the key study procedures and outcome measures}

In order to achieve a high level of standardized processes and collection of data for efficacy and safety endpoints, most of the procedures will be centrally assessed in two CORE-LABs. In each CORE-LAB all examinations will be performed independently by two experienced investigators. The final decisions will be made by consensus. The investigators will be blinded to patient characteristics not related to the index examination. The first CORE-LAB will perform complex analysis (histologic, immunohistochemical, polymerase chain reaction [PCR]) of all collected EMB samples. The second CORE-LAB will perform an analysis of all other relevant procedures: echocardiography, CMR, Holter-ECG, and laboratory tests of all blood and urine samples. Additional blood, urine and stool samples will be stored centrally for future analyses. Before study initiation, all center participants will be verified and trained in performance and submission of high-quality echocardiography and CMR images according to the established unified protocols. 
Table 1. Main inclusion and exclusion criteria.

\section{Main inclusion criteria}

1. Written informed consent to participate in the IMPROVE-MC study.

2. Patient with clinically suspected myocarditis or inflammatory cardiomyopathy (according to the criteria of the ESC Working Group on Myocardial and Pericardial Diseases 2013*); OR/AND,

Patients with already diagnosed active myocarditis (lymphocytic or eosinophilic) or inflammatory cardiomyopathy who will undergo diagnostic right ventricular EMB during the screening.

3. Men or women aged 18-70. Women of childbearing age must have a negative pregnancy test result.

4. No significant improvement in clinical condition or worsening course of the disease despite the standard treatment in the last $\geq 3$ months prior to the screening period.

5. LVEF $\leq 45 \%$ measured by echocardiogram taken during the screening period:

- no significant LVEF improvement in the last $\geq 3$ months prior to the screening period (V1) in the investigator's opinion;

- LVEF should be measured under stable conditions as assessed by the investigator;

- LVEF should be verified in the CORE-LAB.

6. Histologic and immunohistochemical evidence of active myocarditis (lymphocytic or eosinophilic) OR inflammatory cardiomyopathy during the screening period (V1).

7. Absence of cardiotropic viruses in cardiac tissue in PCR analysis during the screening period (V1).

$\geq 1$ clinical presentation and $\geq 1$ diagnostic criteria from different categories; if the patient is asymptomatic

$\geq 2$ diagnostic criteria should be met:

- Clinical presentation with symptoms and signs of myocarditis (chest pain, dyspnea, palpitations etc.);

- Diagnostic criteria:

- any abnormalities in ECG/Holter ECG/stress test;

- elevated troponin concentration;

- structural or functional abnormalities in ECHO/CMR;

- edema or LGE with typical myocarditic pattern on CMR;

- lack of angiographically significant coronary artery disease ${ }^{* *}$ (coronary stenosis $\geq 50 \%$ ) and other causes that could explain the syndrome (hyperthyroidism, congenital heart disease, significant valve disease, etc.).

\section{Main exclusion criteria}

1. Presence of contraindications to immunosuppressive therapy with steroids and/or azathioprine (including hypersensitivity to azathioprine/6-mercaptopurine or prednisone, mainly untreated systemic infection, uncontrolled diabetes, poorly controlled endocrine diseases, osteoporosis, gastric or duodenal ulcer, uncontrolled hypertension, leukocytopenia (leukocyte counts $<4 \times 10^{9} / \mathrm{L}$ ), neutropenia (neutrophils $<1.5 \times 10^{9} / \mathrm{L}$ ), thrombocytopenia (platelet levels $<130 \times 10^{9} / \mathrm{L}$ ), anemia (hemoglobin levels $<11 \mathrm{~g} / \mathrm{dL}$ ).

2. Deficiency or mutation of the enzyme TPMT measured at screening period (V1).

3. Positive test for infections: including HIV, HBV, HCV, tuberculosis (Quantiferon), borreliosis.

4. Another specific cause of heart failure (including severe congenital, valvular, hypertensive, and/or coronary artery disease) that could justify the severity of cardiac dysfunction.

5. Cardiomyopathy based on infiltrative diseases (e.g., amyloidosis), storage diseases (e.g., hemochromatosis, Fabry disease), muscular dystrophies, genetic hypertrophic cardiomyopathy, arrhythmogenic right ventricular cardiomyopathy or known pericardial constriction.

6. Subjects with body mass index $>40 \mathrm{~kg} / \mathrm{m}^{2}$ or body weight $<50 \mathrm{~kg}$.

7. Pregnancy, lactation or women who plan to become pregnant during the trial. Lack of consent to the use of effective forms of contraception.

8. Diagnosed or suspected cardiac sarcoidosis or giant cell myocarditis.

9. Any documented or suspected active malignant neoplasm or history of malignant neoplasm within 5 years prior to the screening period.

10. History of cytostatic therapy or radiotherapy.

11. Liver disease defined as any of the following: AST or ALT or ALP above $3 \times$ ULN; bilirubin $>1.5 \mathrm{mg} / \mathrm{dL}$.

12. Impaired renal function, defined as eGFR $<45 \mathrm{~mL} / \mathrm{min} / 1.73 \mathrm{~m}^{2}$ (CKD-EPI) measured under stable conditions or requiring dialysis.

13. The need or refusal to stop taking any drug considered to interfere with the safe course of the study (e.g., allopurinol).

14. Currently implanted VAD, CRT or heart transplant recipient. 
Table 1 (cont.). Main inclusion and exclusion criteria.

15. Patients with pacemaker or ICD requiring a high percentage of ventricular pacing (>30\%) which could influence the result of LVEF measurement.

16. Gastrointestinal surgery or gastrointestinal disorder that could interfere with trial drug(s) absorption in the investigator's opinion.

17. History or presence of any other disease with a life expectancy $<3$ years.

18. Any contraindications or intolerance to $\mathrm{CMR}^{* * *}$, including but not limited to:

- the presence of cardiac implantable electronic device implanted $<6$ weeks prior;

- pacing capture threshold out of the normal range;

- additional cardiac leads (particularly abandoned pacemaker leads), epicardial leads, fractured leads, additional components such as lead adapters or lead extension;

- aneurysm clips, artificial heart valves, ear implants, or foreign metal objects in the eyes, skin, or body that could be a contraindication to CMR;

- presence of claustrophobia, making it impossible to perform CMR;

- or any other clinical history or study that determines that, in the investigator's judgment, the performance of a CMR may pose a potential risk to the patient.

19. Immunization with live organism vaccines in the last 3 months prior to randomization.

20. Chronic alcohol or drug abuse or non-compliance with medical recommendations or any condition that, in the investigator's opinion, makes patient an unreliable trial subject or be unlikely to complete the trial.

21. Use of other investigational drugs at the time of enrollment, or within 30 days, or within 5 half-lives of enrollment, whichever is longer.

*Definition of clinically suspected myocarditis.

** Lack of angiographically significant coronary artery disease (coronary stenosis $\geq 50 \%$ ) should be confirmed before the current trial using invasive coronary angiography or computed tomography according to the investigator's decision.

${ }^{* * *}$ CMR in non-conditional CIED proved to be safe. CMR in CIED patients will be performed according to HRS 2017 guidelines [27].

ALT - alanine aminotransferase; ALP — alkaline phosphatase; AST — aspartate aminotransferase; CIED - cardiac implantable electronic device; CMR - cardiac magnetic resonance; CRT — cardiac resynchronization therapy; eGFR - estimated glomerular filtration rate;

EMB - endomyocardial biopsy; ESC - European Society of Cardiology; HBV — hepatitis B virus; HCV - hepatitis C virus; HIV - human immunodeficiency virus; HRC - Heart Rhythm Society; ICD - implantable cardioverter-defibrillator; LGE - late gadolinium enhancement; LVEF - left ventricular ejection fraction; PCR - polymerase chain reaction; TPMT — thiopurine S-methyltransferase; ULN — upper limit of normal; VAD - ventricular assist device

Table 2. Reasons for temporal or permanent treatment discontinuation.

The emergence of the following circumstances will require permanent study drug discontinuation:

- Withdrawal of informed consent;

- Patient wish;

- Pregnancy;

- Continuation would be detrimental to the patient's well-being in the investigator's opinion;

- Severe drug(s) related adverse event;

- Significant worsening of symptoms or unacceptable side effects according to the investigator's discretion;

- Other serious conditions limiting further participation in the clinical trial in the investigator's opinion;

- DSMB, SEC or Sponsor's decision to discontinue the study.

The emergence of the following circumstances will require at least temporal study drug discontinuation:

- Moderate-severe infection;

- The need for treatment that is not allowed at the course of the study;

- Three-fold elevation of serum aminotransferase, alkaline phosphatase or amylase above the upper limits of normal;

- White blood cells $<3.8 \times 10^{9} / \mathrm{L}$, neutropenia $\left(<2 \times 10^{9} / \mathrm{L}\right)$, thrombocytopenia $\left(<130 \times 10^{9} / \mathrm{L}\right)$, hemoglobin $\left(<10 \times 10^{9} / \mathrm{L}\right)$;

- Serial reduction of blood cells and/or platelets and/or hemoglobin values within the normal range: e.g., white blood cell count: $6.0 \rightarrow 5.0 \rightarrow 4.5 \times 10^{9} / \mathrm{L}$ over 3 occasions;

- Any protocol deviation that results in a significant risk to the patient's safety.

DSMB — Data Safety Monitoring Board; SEC — Scientific Excellence Committee 


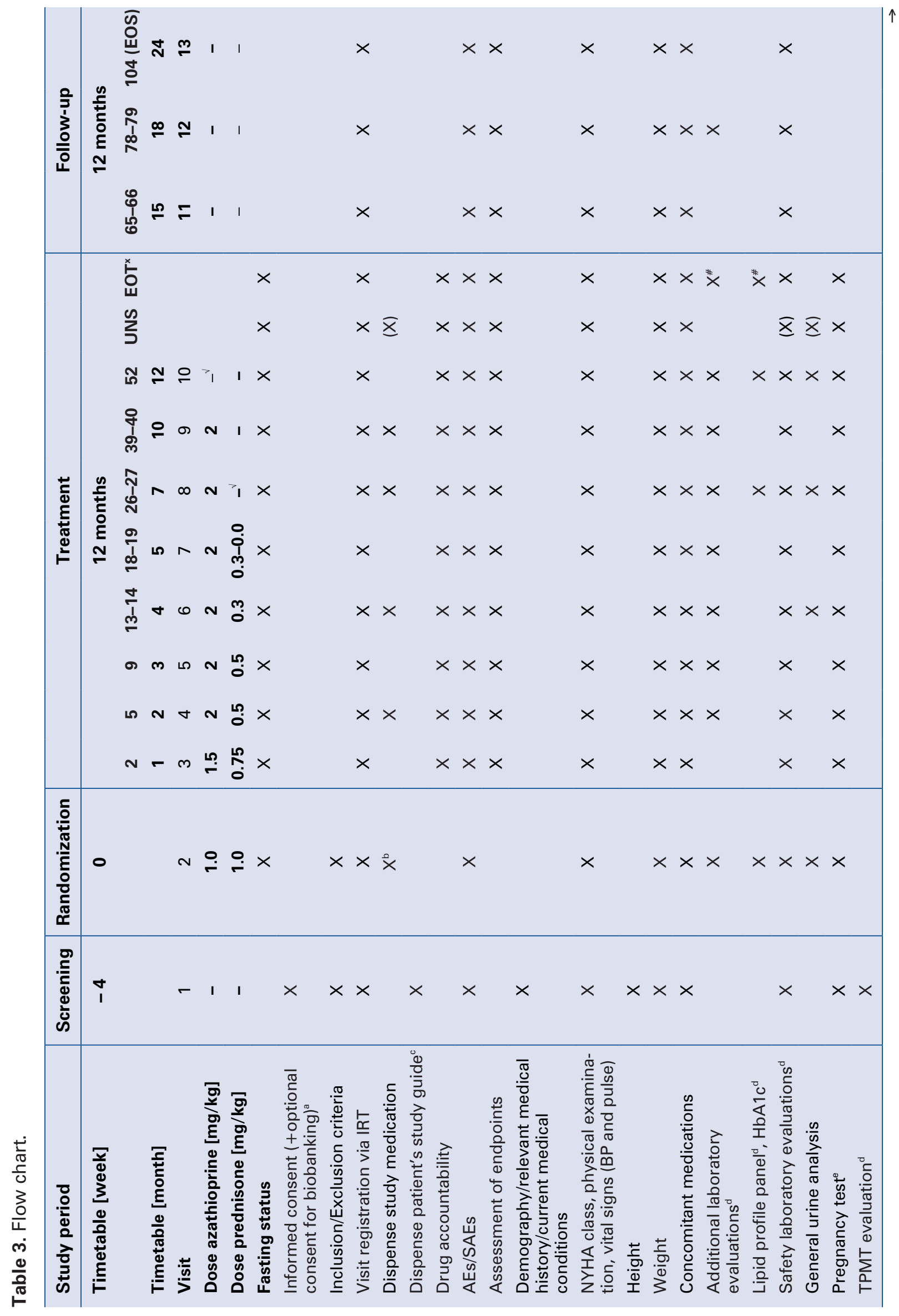




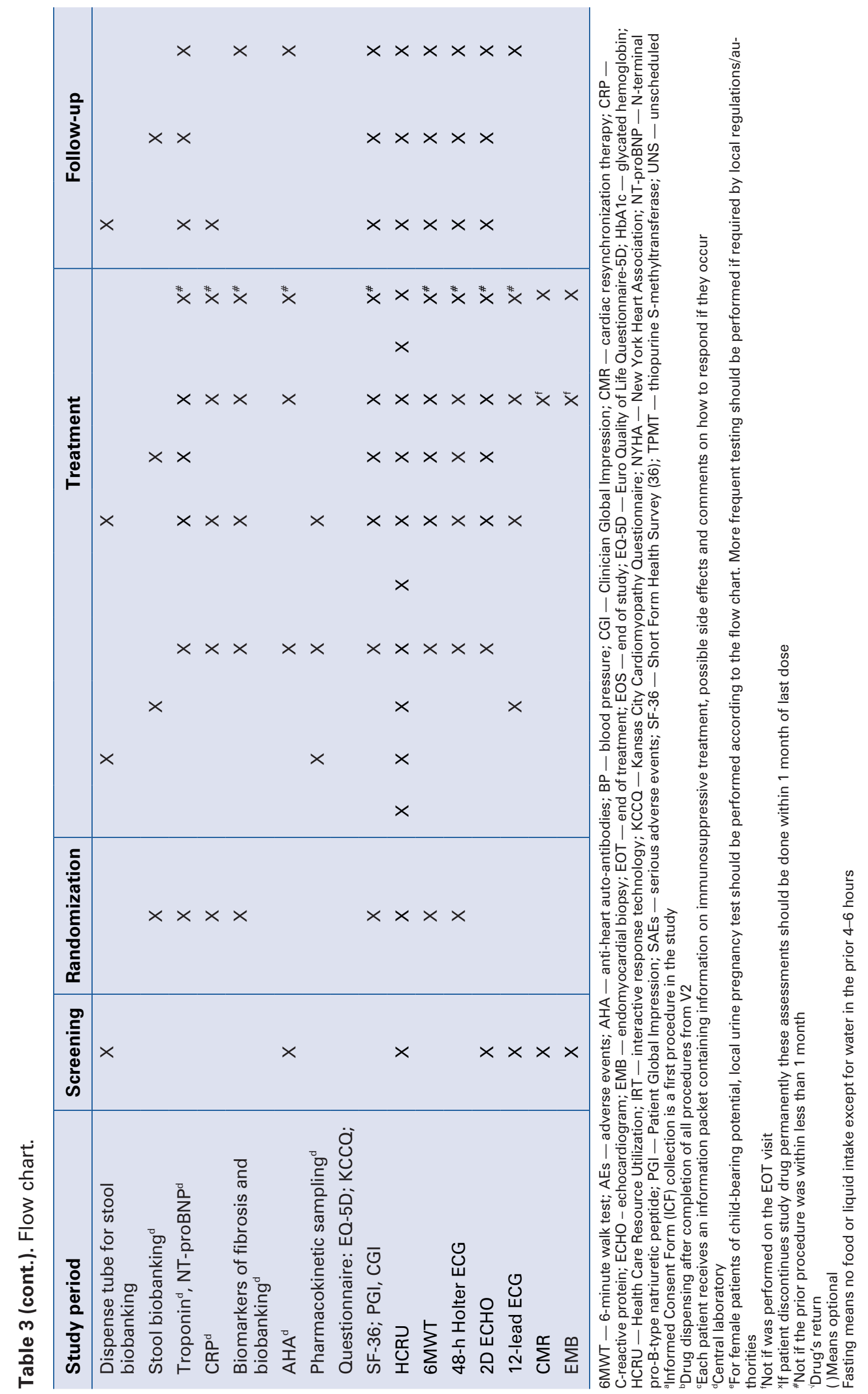




\section{Echocardiography, CMR and 48-hour} Holter ECG

Transthoracic echocardiography will be done at screening to determine eligibility for inclusion in the study. Further echocardiography assessments will be performed at screening and further visits as indicated in the flow chart to investigate the potential effect of the trial medication. All echocardiography images will be transferred to CORE-LAB and, after evaluation, will be sent back to site the decision on patient eligibility for the study (based on LVEF criteria) within $48 \mathrm{~h}$. The images will be assessed using IntelliSpace Cardiovascular and TOMTEC updated software (Philips Medical).

Cardiac magnetic resonance imaging will be performed at screening, and at visit 10 as indicated in the flow chart to investigate a potential effect of the trial medication on cardiac structure and function. CMR protocol will be based on updated Lake Louis Criteria 2018 [20]. All CMR images will be transferred online to CORE-LAB and evaluated using CMR 42 software (GE Medical).

Forty-eight-hour Holter ECGs will be performed at visit 2 (randomization) and further visits in the trial as indicated in the flow chart to investigate the potential effect of the trial medication on type and burden of cardiac arrhythmias. All Holter-ECG examinations will be carried out on the same devices (ECG Lifecard CF), transferred online to CORE-LAB and evaluated using Sentinel software (Reynolds Medical).

\section{Endomyocardial biopsy and analyses of samples}

Endomyocardial biopsy procedure will be performed in all recruiting centers. Routinely EMBs will be performed in the septal region of right ventricle approached by biopsy forceps (Cordis Corp.) from the right femoral or jugular access under fluoroscopic control. Six to 10 right ventricle biopsies (each sample $1-3 \mathrm{~mm}^{3}$ ) are required. Patients will be monitored during and after procedures for possible AEs. EMB procedures will be limited to 2 experienced operators in each center.

All collected biopsy specimens will be promptly immersed in a transport buffer solution in sterile PCR-free tubes and transported on dry ice to the CORE-LAB within $24 \mathrm{~h}$ at $4^{\circ} \mathrm{C}$. Samples will undergo histologic (hematoxylin and eosin [HE] and Masson trichrome) and immunohistochemical, i.e., anti-cluster of differentiation (CD3/CD45/CD68, anti-human leukocyte antigen [HLA] I and II antigens) stainings. Positive immunohistochemistry
EMB finding demonstrating myocardial inflammation will be defined as:

- the presence of CD3-positive lymphocytes $\left(\geq 7\right.$ cells $/ \mathrm{mm}^{2}$ ) and/or CD45-positive lymphocytes $\left(\geq 14\right.$ cells $\left./ \mathrm{mm}^{2}\right)$ and/or CD68-positive cells $\left(\geq 14\right.$ cells $\left./ \mathrm{mm}^{2}\right)$;

- increased expression ( $\geq 2+$; range $0-3$ ) of HLA class I (ABC) and/or class II (DR) antigens;

- and the presence of inflammatory cells adherent to the contour of cardiomyocytes, focally associated with cell necrosis, and depicted by the fraying of sarcolemmal membrane.

In order to verify whether the EMB samples are infected by virus and its type, first hybridization in-situ procedure against eleven virus species will be performed, including the following:

- double strained DNA viruses: adenovirus, cytomegalovirus (CMV), herpes simplex 1 virus, herpes simplex 2 virus, human herpes virus 6 and Epstein-Barr virus (EBV);

- single strained DNA virus: parvovirus B19;

- single strained RNA viruses: enterovirus, hepatitis $\mathrm{C}$ virus (HCV);

- single strained RNA negative-sense viruses: influenza $A$ virus and influenza $B$ virus.

When a positive result by hybridization $i n$-situ reaction will be obtained, additional PCR reaction will be performed to confirm the presence of a detected virus. For quantitative analysis real-time PCR (RT-PCR) reaction will be performed. In the case of parvovirus B19, a negative PCR result will be considered when less than 500 viral copies $/ \mu \mathrm{g}$ genomic DNA will be detected.

All EMB specimens will be stored for possible future analyses.

\section{Safety issues}

At screening patients will be assessed according to the safety checklist to rule out potential contraindications to immunosuppression and to minimize the risk of AEs. Blood will be tested for thiopurine S-methyltransferase (TPMT) deficiency or mutation. Patients with reduced TPMT activity following the administration of thiopurines are at greater risk of AEs.

According to the investigator's decision, patients should also be screened for:

- common latent infections (e.g., hepatitis B virus [HBV], HCV, human immunodeficiency virus [HIV], EBV, CMV, borreliosis, tuberculosis);

- or hidden malignancy (e.g., in situ prostatic, cervical, and/or breast malignancy) — mainly patients aged $\geq 40$ years. 
On all visits, the patient will undergo abbreviated blood tests (assessed in CORE-LAB) and will be asked to report any symptoms of possible infection, HF symptoms, signs of hepatic, renal and/or pancreatic injury. Pregnancy urine testing will be performed in female patients of childbearing potential at all visits before drug(s) re-supply and the patient's continuation in the study.

\section{Outcomes}

The primary endpoint of the study is a change from baseline in LVEF at 12 months. All efficacy and safety endpoints are presented in Table 4. By implementing the described diagnostic and therapeutic regimen, the following short-term and long-term effects are expected:

- improvement of LVEF, cardiac volumes and dimensions;

- reduction of $\mathrm{HF}$ symptoms (improvement in NYHA class);

- improvement of physical performance (increasing the distance in the test 6 -minute walk test);

— improvement in quality of life;

- reduction of the risk of sudden death and HF hospitalization;

- reduction of burden and severity of arrhythmias;

- reduction of the need for heart transplantation, implantation of cardiac devices (i.e., implantable cardioverter-defibrillator [ICD]), ablation of arrhythmia;

- due to the longer (12-month) treatment period, it is expected that the short-term effects will be maintained in a lengthier follow-up;

- pharmacoeconomic benefits.

\section{Data collection and study management}

Interactive response technology will assign random identification numbers to all study participants after being contacted by the investigator. The interactive response technology will specify a unique medication number for the package of the investigational treatment to be dispensed to the patient. The identity of the treatments will be concealed by the use of investigational treatments that are all identical in packaging, labeling, schedule of administration, appearance, taste and odor. Treatment will be blinded to the intervention until the study is completed and the data analyzed.

The study will be supervised by an external Contract Research Organization (CRO). The CRO will monitor the conduct of the trial by regular on-site monitoring visits and in-house data quality review. Data will be collected and stored locally in paper case report form and centrally in electronic case report form provided by CRO. Efficacy, safety, and risk identification will be supervised by the investigators in each center, CRO and Data Safety Monitoring Board. Scientific Excellence Committee consisting of independent international experts will be established to support the scientific and advisory function of the trial. The study will involve a multidisciplinary team consisting of cardiologists, radiologists, clinical immunologists and pathologists, working together to deal with the most demanding cases and to make the most appropriate decisions to ensure the highest patient safety and optimization of the effectiveness of the treatment. In order to maintain safety of the therapy, the study participants will have regular medical examinations (i.e., assessment of the severity of HF symptoms), physical examination (with assessment of vital signs, body weight measurement), laboratory blood and urine tests, and additional procedures, as shown in Table 3. An additional important element of the study will be the assessment of the quality of life, done using standardized quality of life tests. At each visit, the patient will be instructed and educated about the rules of taking medications, the principles of a healthy and hygienic lifestyle, possible restricted drugs. The patient will have $24 \mathrm{~h} / 7$ contact with a member of the research team throughout the study.

Details on the study procedures, administrative structure, and conduct of the trial are presented in the complete study protocol.

\section{Statistical methods}

All statistical analyses will be performed by independent, external statisticians. Per-protocol, intention-to-treat and safety sets will be used for statistical analyses, according to the trial statistical analysis plan designed by CRO. For all endpoints, the superiority of immunosuppressive treatment vs. placebo will be evaluated with a two-sided test. There has been no study evaluating the effectiveness of immunosuppressive treatment in 1-year follow-up, therefore based on previous studies, it is currently estimated that approximately 46 patients per group are required for the primary endpoint analysis, including $5 \%$ margin of error, test power 0.8 , standard deviation of LVEF $13 \%$, and $20 \%$ of patients lost during follow-up. The sample size for the key-secondary endpoints is covered with the 46 patients per group. The Data Monitoring Committee with $\mathrm{CRO}$ will monitor safety and efficacy data. 
Table 4. Main study efficacy and safety endpoints.

\section{Primary endpoint}

— Change from baseline in LVEF at 12 months

\section{Key secondary endpoints}

- Proportion of patients who responded to immunosuppressive therapy as defined by an LVEF absolute increase of $\geq 10 \%$ over time

— Change in the LV end-systolic and end-diastolic dimensions as well as the LV end-systolic and end-diastolic volumes over time

- Change from baseline in NYHA class over time

- Occurrence of adjudicated HF decompensation (hospitalization or ambulatory visit)

Further secondary endpoints

— Change from baseline in percentage of patients in NYHA III/IV and NYHA II class over time

- Occurrence of need for diuretic (intravenous administration)

- Change from baseline in the 6-minute walk test distance over time

- Time to first adjudicated hospitalization for HF

- Time to first all-cause hospitalization

- Occurrence (first and recurrent) of all-cause hospitalization, HF hospitalization, HF outpatient visit, myocarditis or inflammatory cardiomyopathy recurrence, all-cause death, heart transplantation, implantation of cardiac device (pacemaker, ICD, CRT, VAD) assessed in combination or independently

- New onset AF

- New onset sustained VT or VF

$-\geq 50 \%$ reduction from baseline in VEBs number in $48 \mathrm{~h}$ Holter monitoring over time

$-\geq 50 \%$ reduction from baseline in nonsustained VT number in $48 \mathrm{~h}$ Holter monitoring over time

$-\geq 50 \%$ reduction from baseline in AF burden in $48 \mathrm{~h}$ Holter monitoring over time

- Changes from baseline in echocardiographic parameters (left and right ventricular function; dimensions and volumes of the heart chambers; thickness of myocardial walls; tissue Doppler velocity of the mitral annulus; strain and strain rate imaging changes) over time

- Changes from baseline in CMR results (EGE, LGE, edema, LV dimensions and volumes, T1/T2 mapping) after 1-year

- Changes from baseline in concentration of biomarkers of fibrosis and myocardial necrosis (troponin I, NT-proBNP, sST2, galectin-3) over time

- Changes from baseline in concentration of anti-heart auto-antibodies over time

- Qualitative and quantitative change from baseline in inflammatory infiltration, HLA expression and fibrosis in EMB after 1-year

- Change from baseline of patients' health status as assessed by the patients self-reported EQ-5D over time

- Change from baseline in clinical summary score (HF symptoms and physical limitations domains) of KCCO over time

- Change from baseline in $\mathrm{KCCQ}$ overall summary score over time

- Change from baseline in KCCQ total symptom score over time

- Change from baseline in $\mathrm{KCCO}$ individual domains over time

- Change from baseline in KCCQ based on patient-preferred outcome over time

- Change from baseline in SF-36 questionnaire overall summary score over time

- Change from baseline in PGI-I scale over time

- Change from baseline in CGI-I scale over time

— Change from baseline in health economic analysis by HCRU

- Cost-effectiveness analysis

Secondary endpoints will be assessed in 3-month intervals up to 24-month from the randomization (unless otherwise indicated). 
Table 4 (cont.). Main study efficacy and safety endpoints.

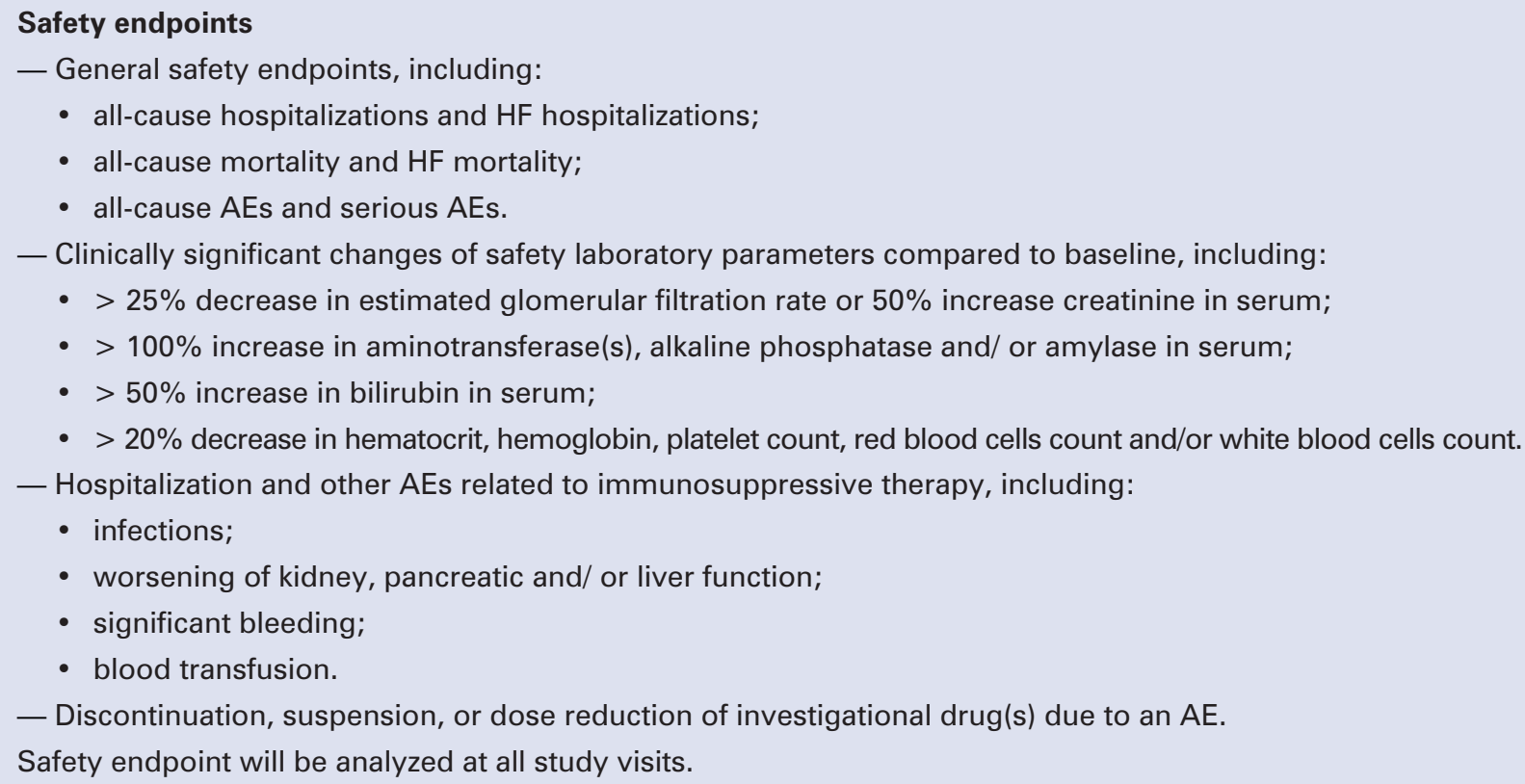

AEs - adverse events; AF — atrial fibrillation; CGI-I — Clinician Global Impression of Improvement; CRT — cardiac resynchronization therapy; EGE — early gadolinium enhancement; EMB — endomyocardial biopsy; EQ-5D — Euro Quality of Life Questionnaire-5D; HCRU — Health Care Resource Utilization; HF — heart failure; HLA — human leukocyte antigen; ICD — implantable cardioverter-defibrillator; KCCQ — Kansas City Cardiomyopathy Questionnaire; LGE — late gadolinium enhancement; LV — left ventricle; LVEF — left ventricular ejection fraction; NT-proBNP - N-terminal pro-B-type natriuretic peptide, NYHA - New York Heart Association; PGI-I — Patient Global Impression of Improvement; SF-36 — Short Form Health Survey (36); sST2 — soluble interleukin-1 receptor-like 1; VAD — ventricular assist device; VEBs — ventricular ectopic beats; VT — ventricular tachycardia

\section{Ethics and dissemination}

The trial will be performed in compliance with the protocol, the ethical principles in full conformity with the Declaration of Helsinki, in accordance with the ICH Harmonized Guideline for Good Clinical Practice and Standard Operation Procedures, and other relevant regulations. The study protocol and written consent form have been approved by the Research Ethics Board of the Medical University of Warsaw (KB/164/2020). Participants will be fully informed about the study, and informed consent will be obtained (including optional consent for biobanking). Any modifications of the protocol that might impact patient safety, confidentiality, or other significant conduct of the study will be submitted to and reviewed by the Research Ethics Board and Office for Registration of Medicinal Products, Medical Devices and Biocidal Products [21] before implementation. The manufacturers of the investigated medicinal products will have no role in the trial design, conduct of the study, or in the analysis or interpretation of the data. The findings of this study, whether positive or negative, will be published in a peer-reviewed journal in accordance with CONSORT [22]. Access to the full protocol, participant-level dataset, and statistical code may be granted upon request.

\section{Discussion}

Due to the fact that currently there is no evidence-based consensus on the treatment of myocarditis and/or inflammatory cardiomyopathy and the strategies used are unsatisfactory, the results of this study are highly desirable for medical and ethical reasons. Based on the mechanisms of action of the investigated drugs and the results of previous trials [23, 24], it is assumed that patients with myocarditis and/or inflammatory cardiomyopathy should benefit from combined treatment with prednisone and azathioprine on top of guideline-directed therapies. Investigators will also be encouraged to treat participants to the best standard of care in compliance with the local guidelines and recommendations for treatment of $\mathrm{HF}$ and comorbidities. Although there are preliminary data that immunosuppressive treatment might be beneficial irrespectively of initial LVEF (even in patients with preserved LVEF) [25], it was decided to include patients with $\mathrm{LVEF} \leq 45 \%$ as the 
population most likely to benefit from the therapy, and in order to achieve statistical significance of the results. Virus-positive patients detected on EMB will be excluded from the study according to the current ESC consensus statement [1]. To increase detectability of immune-mediated cases of myocarditis or inflammatory cardiomyopathy anti-heart auto-antibodies will be searched for in the sera. There is strong data that in anti-heart auto-antibodies-positive patients immunosuppressive and/or immunomodulative treatment may be particularly beneficial [26].

The duration of the active treatment was based on pharmacokinetic and complementary properties of the studied medications. Prednisone will be started with high doses for initial suppression of the immune system, while a suppressive effect of azathioprine may not become apparent until after several weeks or months of treatment. It seems that azathioprine should be used for at least 6 months and it is crucial for maintaining the effects of therapy and to prevent relapses after premature stopping of the treatment. This was also the reason for the prolongation of the active treatment period to 12 months. A subsequent 12 -month follow-up period will allow for the observation of potential relapses in the active-treatment group, as well as spontaneous recoveries in the placebo group.

The overall benefits and safety profile of prednisone and azathioprine have been confirmed in multiple clinical trials and years of observation, mainly in patients with organ transplant, inflammatory or autoimmune diseases. Both drugs are registered and approved in other indications than the current one (myocarditis and/or inflammatory cardiomyopathy) in the proposed clinical trial. The safety profile of prednisone and azathioprine in the study group should follow a similar trend that was previously observed in Frustaci et al. [23] and Wojnicz et al. [24] studies. Safety will be ensured by close monitoring of the subjects for AEs with both, clinically and laboratory testing.

It is expected that the IMPROVE-MC study should confirm available preliminary results by providing high-quality (and easily implemented) results on the efficacy and safety of the therapeutic scheme, which will impact international guidelines for the treatment of myocarditis and/or inflammatory cardiomyopathy.

\section{Funding}

Non-commercial study. The trial sponsor is the Medical University of Warsaw (Poland). The study is supported by a grant (12 $832586.40 \mathrm{PLN}$; approx. $3 \mathrm{mln}$. Euro) from the Polish Medical Research Agency (grant number: 2019/ABM/01/00047-00).

\section{Conflict of interest: None declared}

\section{References}

1. Caforio ALP, Pankuweit S, Arbustini E, et al. Current state of knowledge on aetiology, diagnosis, management, and therapy of myocarditis: a position statement of the European Society of Cardiology Working Group on Myocardial and Pericardial Diseases. Eur Heart J. 2013; 34(33): 2636-48, 2648a, doi: 10.1093/ eurheartj/eht210, indexed in Pubmed: 23824828.

2. Lynge TH, Nielsen TS, Gregers Winkel Bo, et al. Sudden cardiac death caused by myocarditis in persons aged 1-49 years: a nationwide study of 14294 deaths in Denmark. Forensic Sci Res. 2019; 4(3): 247-256, doi: 10.1080/20961790.2019.1595352, indexed in Pubmed: 31489390.

3. Anzini M, Merlo M, Sabbadini G, et al. Long-term evolution and prognostic stratification of biopsy-proven active myocarditis. Circulation. 2013; 128(22): 2384-2394, doi: 10.1161/CIRCULATIONAHA.113.003092, indexed in Pubmed: 24084750.

4. Fabre A, Sheppard MN. Sudden adult death syndrome and other non-ischaemic causes of sudden cardiac death. Heart. 2006; 92(3): 316-320, doi: 10.1136/hrt.2004.045518, indexed in Pubmed: 15923280.

5. Tymińska A, Ozierański K, Caforio ALP, et al. Myocarditis and inflammatory cardiomyopathy in 2021: an update. Pol Arch Intern Med. 2021; 131(6): 594-606, doi: 10.20452/pamw.16010, indexed in Pubmed: 34057322.

6. Gore I, Saphir O. Myocarditis. Am Heart J. 1947; 34(6): 827-830, doi: 10.1016/0002-8703(47)90147-6.

7. Basso C, Calabrese F, Corrado D, et al. Postmortem diagnosis in sudden cardiac death victims: macroscopic, microscopic and molecular findings. Cardiovasc Res. 2001; 50(2): 290-300, doi: 10.1016/s0008-6363(01)00261-9, indexed in Pubmed: 11334833.

8. Mason JW, O'Connell JB, Herskowitz A, et al. A clinical trial of immunosuppressive therapy for myocarditis. The Myocarditis Treatment Trial Investigators. N Engl J Med. 1995; 333(5): 269275, doi: 10.1056/NEJM199508033330501, indexed in Pubmed: 7596370.

9. Felker GM, Hu W, Hare JM, et al. The spectrum of dilated cardiomyopathy. The Johns Hopkins experience with 1,278 patients. Medicine (Baltimore). 1999; 78(4): 270-283, doi: 10.1097/00005792199907000-00005, indexed in Pubmed: 10424207.

10. Ponikowski P, Voors AA, Anker SD, et al. 2016 ESC Guidelines for the diagnosis and treatment of acute and chronic heart failure: The Task Force for the diagnosis and treatment of acute and chronic heart failure of the European Society of Cardiology (ESC)Developed with the special contribution of the Heart Failure Association (HFA) of the ESC. Eur Heart J. 2016; 37(27): 2129-2200, doi: 10.1093/eurheartj/ehw128, indexed in Pubmed: 27206819.

11. Heymans S, Eriksson U, Lehtonen J, et al. The Quest for New Approaches in Myocarditis and Inflammatory Cardiomyopathy. J Am Coll Cardiol. 2016; 68(21): 2348-2364, doi: 10.1016/j. jacc.2016.09.937, indexed in Pubmed: 27884253.

12. Dominguez F, Kühl U, Pieske B, et al. Update on myocarditis and inflammatory cardiomyopathy: reemergence of endomyocardial biopsy. Rev Esp Cardiol (Engl Ed). 2016; 69(2): 178-187, doi: 10.1016/j.rec.2015.10.015, indexed in Pubmed: 26795929. 
13. Cooper LT, Berry GJ, Shabetai R. Idiopathic giant-cell myocarditis--natural history and treatment. Multicenter Giant Cell Myocarditis Study Group Investigators. N Engl J Med. 1997; 336(26): 1860-1866, doi: 10.1056/NEJM199706263362603, indexed in Pubmed: 9197214.

14. Sinagra G, Anzini M, Pereira NL, et al. Myocarditis in clinical practice. Mayo Clin Proc. 2016; 91(9): 1256-1266, doi: 10.1016/j. mayocp.2016.05.013, indexed in Pubmed: 27489051.

15. Azathioprine. http://leki.urpl.gov.pl/files/43_Azathioprine_VIS_ tabl_50mg.pdf.

16. Azathioprine. https://www.uptodate.com/contents/azathioprine-drug-information? topicRef $=127933 \&$ source $=$ see link.

17. Encorton. http://leki.urpl.gov.pl/files/24_Encorton.pdf.

18. Chan AW, Tetzlaff JM, Gøtzsche PC, et al. SPIRIT 2013 explanation and elaboration: guidance for protocols of clinical trials. BMJ. 2013; 346: e7586, doi: 10.1136/bmj.e7586, indexed in Pubmed: 23303884.

19. Reed JL, Pipe AL. The talk test: a useful tool for prescribing and monitoring exercise intensity. Curr Opin Cardiol. 2014; 29(5): 475-480, doi: 10.1097/HCO.0000000000000097, indexed in Pubmed: 25010379.

20. Ferreira VM, Schulz-Menger J, Holmvang G, et al. Cardiovascular magnetic resonance in nonischemic myocardial inflammation: expert recommendations. J Am Coll Cardiol. 2018; 72(24): 3158-3176, doi: 10.1016/j.jacc.2018.09.072, indexed in Pubmed: 30545455.

21. http://www.urpl.gov.pl/en.

22. Ioannidis JPA, Evans SJW, Gøtzsche PC, et al. Better reporting of harms in randomized trials: an extension of the
CONSORT statement. Ann Intern Med. 2004; 141(10): 781-788, doi: 10.7326/0003-4819-141-10-200411160-00009, indexed in Pubmed: 15545678.

23. Frustaci A, Russo MA, Chimenti C. Randomized study on the efficacy of immunosuppressive therapy in patients with virus-negative inflammatory cardiomyopathy: the TIMIC study. Eur Heart J. 2009; 30(16): 1995-2002, doi: 10.1093/eurheartj/ ehp249, indexed in Pubmed: 19556262.

24. Wojnicz R, Nowalany-Kozielska E, Wojciechowska C, et al. Randomized, placebo-controlled study for immunosuppressive treatment of inflammatory dilated cardiomyopathy: two-year follow-up results. Circulation. 2001; 104(1): 39-45, doi: 10.1161/01. cir.104.1.39, indexed in Pubmed: 11435335.

25. De Luca G, Campochiaro C, Sartorelli S, et al. Efficacy and safety of mycophenolate mofetil in patients with virus-negative lymphocytic myocarditis: a prospective cohort study. J Autoimmun. 2020; 106: 102330, doi: 10.1016/j.jaut.2019.102330, indexed in Pubmed: 31488318.

26. Caforio AL, Cheng C, Perazzolo Marra M, et al. How to improve therapy in myocarditis: role of cardiovascular magnetic resonance and of endomyocardial biopsy. Eur Heart J Suppl. 2019; 21(Suppl B): B19-B22, doi: 10.1093/eurheartj/suz014, indexed in Pubmed: 30948937.

27. Indik JH, Gimbel JR, Abe H, et al. 2017 HRS expert consensus statement on magnetic resonance imaging and radiation exposure in patients with cardiovascular implantable electronic devices. Heart Rhythm. 2017; 14(7): e97-e9e153, doi: 10.1016/j. hrthm.2017.04.025, indexed in Pubmed: 28502708. 